\title{
Wave/Particle Duality and Biomedical Research Designs
}

\section{Sarah S. Knox}

Department of Epidemiology, School of Public Health, Member WVU Cancer Institute, West Virginia University, USA

*Corresponding author: S Knox, Professor of Epidemiology, School of Public Health, Member WVU Cancer Institute, West Virginia University, USA, Tel: 304-293-1058; E-mail: sknox@hsc.wvu.edu

Received date: July 03, 2018; Accepted date: July 10, 2018; Published date: July 14, 2018

Copyright: $\odot 2018$ Knox SS. This is an open-access article distributed under the terms of the Creative Commons Attribution License, which permits unrestricted use, distribution, and reproduction in any medium, provided the original author and source are credited.

\begin{abstract}
Scientific literature that directly addresses the underlying framework or paradigm of biomedical research is sparse. We consider ourselves objective scientists in well-defined scientific disciplines and design our studies based on state-of-the-art evidence in our respective fields. However, there is a pervasive underlying assumption that remains unexamined, and in this author's view scientifically unjustified. It is the assumption that because medical conditions occur in the physical body, their causality must be traceable to some form of particulate matter. Although this unspoken assumption is axiomatic in current research, it lacks supporting data. The thesis of this paper is that our assumptions about the underlying nature of reality are outdated with respect to modern physics. Because these assumptions serve as the basis for our study designs, i.e., determine the methodology as well as the variables we include and ignore, inaccurate assumptions risk excluding important data and biasing conclusions.
\end{abstract}

Evidence based medicine is only as good as the research designs on which the evidence is based. If data are lacking because a question, or part of the data related to the question, have been ignored, then the 'evidence' is incomplete. What is missing in mainstream biomedical research, particularly in cancer, is the important role played by endogenously generated bioelectric fields in molecular, cellular and systemic signal processing.

\section{Wave/Particle Duality}

A fundamental principle of quantum mechanics holds that at a subatomic level, a physical system such as an electron exists partly and simultaneously in all its theoretically possible states until it is measured or observed. At the time of measurement, the wave function (as defined by the Schrodinger equation) collapses, resulting in only one of the possible configurations (e.g., wave or particle). From a standpoint of mathematics, this is because the Schrodinger equation is deterministic and probabilistic, being based on information from initial conditions and the possibility of multiple potential pathways between the initial condition and the end measurement. The probabilistic nature of the outcome is due not to imprecise measurement technique but to the nature of reality. In order for a particle to have a definite position, it must be measured while still. To have a specific momentum, it must be in motion, which means that the position and the momentum cannot be measured simultaneously. The same concept applies to measuring waves. According to quantum mechanics, light can consist of either particles (photons) or waves, depending on how the measurement is performed. The essence of this concept is that you cannot observe waves and particles in the same measurement, so the way the experiment is designed determines the outcome. Expressed differently, at a quantum level what exists are not bits of matter but patterns of probability. According to Werner Heisenberg, "all particles are made of the same fundamental substance, which can be designated energy or matter; or we can put things as follows: the basic substance 'energy' becomes 'matter' by assuming the form of an elementary particle [1]." This theoretical interpretation which directly contradicts the assumption of primary substance as matter, is controversial and still being debated by quantum theorists. However, on a practical level, the utility of quantum mechanics has been demonstrated by the number of useful products it has contributed to our daily lives, including semiconductors, transistors, quantum thermometers, lasers, and fibre optics.

The concept of wave/particle duality is often illustrated by what is known as the 'double-split' experiment. If single electrons are propelled one at a time through a barrier with two slits, an observer placed directly on the other side of this barrier will observe single electrons going through either one slit or the other. This is what we would expect from the behaviour of particles based on Newtonian mechanics. However, if electrons continue to be projected through the two-slit barrier one-at-a-time onto a screen placed beyond it (equivalent to a different observer), over the course of time the projected pattern will be an interference pattern typical of waves. Although logic tells us that an electron can only enter one of two slits and that the pattern projected onto the screen should therefore be two stripes, what we see is a pattern of multiple stripes. Multiple stripes would be the expectation only if waves had gone through the slits causing interference patterns that either reinforce or cancel each other out. These, seemingly contradictory results based on the type of observation, are not the result of poor measurement technique but a reflection of the nature of reality at a subatomic level, where matter and energy are two aspects of the same underlying reality.

Although the concept of wave/particle duality is counterintuitive and its implications still under debate by quantum theorists, its utility and value on an applied level are not. Despite its accuracy, the implications of quantum mechanics for molecular biology and biomedical research designs have not been recognized or utilized. The way we set up our experiments determines whether we see a 'wave' or a 'particle, i.e., energy or matter; and mainstream biomedicine is currently setting up experiments to see only particles (e.g., targeted therapies in cancer). 


\section{DNA: Wave or Particle?}

An excellent example of this is the way we determine the characteristics of DNA. The current protocol involves separating the two strands of the double helix to access and measure the sequence of base pairs. These sequencing methods and their offshoots (e.g., AFEAP cloning [2], multiple-site fragment deletion, insertion and substitution [3], and restriction-free reconstitution using single-primer PCRs [4]), are continually improving in speed and efficiency, and have provided a great deal of very useful information about genetic risks and Mendelian inheritance, even facilitating the laboratory synthesis of DNA. They have also contributed to the relatively new field of epigenetics, which has demonstrated that genes often have multiple functions and that the characteristic properties a gene expresses at a particular moment in time are influenced by factors in its surrounding microenvironment other than base pair sequence. Epigenetic studies have shown that the flexibility in gene expression facilitated by microenvironmental processes such as methylation, acetylation, phosphorylation [5,6], can facilitate flexibility in function and accommodate continual dynamic changes in physiological needs. With respect to wave/particle duality, sequencing methods highlight particulate aspects of DNA, but obscure another characteristic function of the DNA strand, which is that of an electrical conductor [7-9]. DNA is a highly charged molecule that carries two ionized monovalent groups on its outer surface per base pair [10]. In order to see the 'wave form', i.e., the electrical conductance, the double helix must be left intact. The charge conduction pathway consists of wave functions that extend perpendicular to the base planes, overlapping with neighbouring planes to form a $\pi$-orbital system [11]. The biomedical research community seems completely unaware that the way they set up their experiments determines which aspects of DNA they see. Most scientists are unaware that DNA has any characteristics other than those of particulate matter. The electronic properties of DNA, like its particulate characteristics, are sensitive to epigenetic changes in the surrounding microenvironment that can modify its phenotype from a conductor to an insulator [11].

\section{Endogenous Bioelectric Signalling at the Cellular Level}

The relevance of quantum mechanics for biomedical research lies in the implications for study design. Since every quantic entity can be described partly in terms of both particles and waves, at a subatomic level there is no difference between matter and force field. Biophysics and biochemistry are two sides of the same coin. In fact, one of the physical characteristics of DNA that prevents it from being activated inappropriately is an attraction between the negatively charged phosphate in the DNA backbone and the positively charged lysine tail of the histones around which the DNA is coiled [12]. Neutralization of this charge (e.g., by acetylation) is part of the epigenetic process of activating gene expression.

The quantum conundrum is that it is not possible to identify the electrical characteristics of DNA by separating the double helix, nor is it possible to identify the particulate aspects of nucleotides by leaving the helix intact. This is a direct consequence of wave/particle duality. If we set up the experiment one way (sequencing), we see particles and if we set it up another way, we see waves (conductance), but we cannot see both with the same experiment. No matter how advanced the technology or how accurately we measure, if our hypotheses about causality determine our research designs and the research designs set up the experiments to see only one type of data, bias has been built into the experiment. The choice of setting up the experiment to see waves or particles is one that most scientists are currently making unawares. Biomedical research tends to focus on particles (e.g., base pairs, mutations, proteins, etc.) to the exclusion of waves (endogenous bioelectric fields). Well-known examples of endogenously generated fields are electrophysiological measurements such as EEG, galvanic skin response, electrocardiograms, and muscle tension, all of which can be measured by electrodes placed on the skin. However, these signals that are used to diagnose underlying pathology (e.g., myocardial infarction), are routinely regarded as epiphenomena or biomarkers that have no causal role. This interpretation stems from the underlying belief that causality can be found only in matter.

Many aspects of the cell cycle and systemic functioning are regulated by biophysical (bioelectric) signals. These include cell division and proliferation, embryonic development (e.g., left-right body asymmetry, axon outgrowth), epithelial wound healing, tissue regeneration and cancer cell migration [13-15]. These signals stem from the fact that all cells (not just neurons) receive and respond to both bioelectric and biochemical cues [16]. There are two cellular mechanisms in somatic cells that are primarily responsible for the generation of these endogenous bioelectric fields. The first involves cell and mitochondrial membranes that contain multiple types of ion channels (e.g., $\mathrm{K}+, \mathrm{Na}+, \mathrm{Cl}-$ ) which create concentration gradients across the membrane and function as gates for substances that enter and exit the cell. Ions cross the membrane by diffusion and by electric fields. These gradients create a voltage potential across the membrane, which in healthy cells is more negative on the inside of the cell than on the outside [13,17-22]. Eukaryotic cells also have a cytoskeleton composed primarily of microtubules and actin filaments that structure the cell and generate movements without any major chemical changes. These hollow microtubules that play a very important role in cell division vibrate and have electric dipole moments due to asymmetry in their electron distribution [23-25]. Their structure and vibrational characteristics create an ionic charge layer on the outside of the microtubule. These bioelectric mechanisms, as well as the polar and polarization properties of matter in general, have led to the conclusion that endogenous electromagnetic fields play an important role in organization and order $[13,26]$.

\section{Limitations of Particulate Matter Study Designs in Cancer}

The primary objective of cancer therapeutics is to kill cancer cells; and this was the justification for the use of cytotoxic chemotherapies that inhibit mitosis in all cells, because they are particularly toxic to cancer cells (which divide much more rapidly than normal cells). However, in order to reduce collateral damage, therapeutics in recent years have evolved towards pharmaceutical treatments that inactivate specific molecular targets $[27,28]$. The aim of targeted treatments is to knock out key biochemical signalling pathways relevant to cancer phenotypes using targets such as the inhibition of receptor tyrosine kinases that regulate different cellular processes (e.g., growth promoting signals from hormones in certain types of breast cancer).

Despite these advances and increasing knowledge of tumourbiology, and despite initial positive responses to therapy, cancers seem to have a virtually limitless ability to respond to treatment by reprogramming cells and developing resistance to specific therapeutics [29]. In fact, "oncology has one of the poorest records for investigational drugs in clinical development, with success rates that are more than three times lower than for cardiovascular disease" [29] and cancer chemoprevention is "an almost universal failure" [30]. 
Although this sounds dire, therapeutic progress has been made. Overall age adjusted cancer mortality rates decreased about $1.5 \%$ per year between 2003-2012 [31]. Despite this fact, mortality rates have risen for certain cancers, such as liver, anus, and pancreatic cancer in both sexes. In men, mortality rates for melanoma, tonsil, thyroid and oropharynx cancers have risen, as have uterine and vulvar cancers in women [31]. Thus, we have made progress but it has not kept pace with the rapid technical advances and large financial investments in omits and analytic technologies.

There are multiple possible explanations for this, one of which may be an almost exclusive emphasis on particulate matter (biochemical) aspects of cancer. The current paradigm for cancer research is a cell centric model based on the clonal expansion and selective growth of specific gene mutations or expression profiles [32]. However, tumours of similar phenotypes, including cells within the same tumour, display a remarkable heterogeneity with respect to mutated genes [32]. Additionally, the number of mutations that occur on a daily basis far exceeds the incidence or prevalence of cancer and does not lend itself to a reductionist model. Cells wear out and are replaced on a regular basis, which means that the probability of mutations is extremely high. It has been estimated that of the 10 trillion cells in the human body, about 70 billion divide to make new cells every day [33] and that the number of DNA lesions per day lies between 104 and 105 [34, 35]. Mutations can come about through depurination and depyrimidation of DNA; proofreading and mismatch errors during DNA replication; deamination of 5-methylcytosine to produce $\mathrm{C}$ to $\mathrm{T}$ base pair substitutions; and damage to DNA or its replication by cellular defence mechanisms [36]. In fact deamination alone, is estimated to occur at steady-state level of 5-10,000 lesions per cell per day, which if not corrected would be mutagenic $[37,38]$. If random mutations were enough to cause cancer, there is a high probability that human evolution would have gone the way of the dinosaurs.

It has also been demonstrated that genome-wide epigenetic changes actually precede cancer [39], indicating the carcinogenic involvement of gene activity not related to mutations. Genome-wide changes also suggest disruption and/ or malfunction in multiple systems that could destabilize anti-tumour defences and create host susceptibility to tumorigenesis at a localized site, (e.g., by reducing DNA repair capability, apoptosis or immune defences)

Biological signalling is the method the body uses to regulate and coordinate the activity of interacting systems and maintain dynamic equilibrium at different organizational levels. The focus of current research in this area is biochemistry, and a key part of this is the conceptualization of regulatory pathways as simple linear chains of causation that lead to a particular phenotype; whereas, in reality, these pathways fan out to affect hundreds to thousands of other genes [40]. This means that there can be unintended off-target effects even with targeted therapies [41]. The ca. 20,000 protein coding genes in the human genome interact with networks of transcription factors and other gene expression regulators (e.g., RNA binding proteins, mi RNAs, chromatin), to give rise to gene expression patterns that are highly complex [39]. This complexity takes the form of molecular interactions between genes and proteins that are regulated by networks rather than a series of parallel pathways [27, 42-44]. This signalling includes fulfilling energy requirements and metabolic changes required by abnormal cell proliferation in cancer, as well as generating biosynthetic activity that greatly differs from that of normal cells [45].

For these reasons, and the fact that tumour cells can be reverted to normal cells when placed into different microenvironments
[46-48], many scientists have begun to refer to cancer is a systemic disease and to tumours as dynamic organisms involving complex interactions and dysfunction (not just mutations) at multiple levels [27, 49-52].

\section{Implications of Study Design for Clinical Inference in Oncology}

These data lead us to the conclusion that neither the clonal genetic model nor the epigenetic progenitor models are sufficient for understanding cancer etiology and progression. Both are devoid of any discussion of the body's pervasive bioelectric signalling systems. The importance of wave/particle duality for design in biomedical research is illustrated by the fact that bioelectric cell states such as those described above, result from post-translational gating of ion channels (their being opened and closed by a range of local but non-cellautonomous signals) [53]. This means that two cells with the same protein profile can have vastly different physiological states [53]. Consequently, proteomics (particulate matter) analyses are not sufficient for understanding their function. This information is unknown to mainstream oncology researchers because their experimental designs do not make it available. The fact that bioelectric signalling can induce or suppress tumorigenesis without DNA damage through modulation of a number of different channels or pumps [53] is also unrecognized by most medical scientists. Yet accumulating data indicate that In order to understand abnormal cell proliferation and metastasis, we need to measure both bioelectric and biochemical signalling and integrate them into a cohesive conceptualization of the dynamics in tumour biology.

"Biologists are beginning to explore the idea that cancer is not a genetic disease of specific loci but rather a kind of attractor in a multidimensional transcriptional space describing cell states" [53]. This refers to states of dynamic equilibrium to which fluctuating systems return after being perturbed. The human body, which functions far from thermodynamic equilibrium, is a dynamical system that exchanges air, nutrients and other substances with the surrounding environment. To maintain health, the body must be facile at continually responding to internal and external demands and stimuli. Each cell in the body has essentially the same genes. Differentiation into phenotype and maintenance of functional equilibrium require coordination of gene and protein expression within and between various tissues, as well as the direction of blood, energy and other functions to locations where they are needed at a particular point in time. All of this requires the transmission and coordination of information through multiple hierarchical systems, and occurs through signalling processes characterized by both wave and particulate aspects. The primary difference is that biochemical signals are transferred from receptor to receptor, whereas biophysical (bioelectric) signals are propagated as fields.

Attractors return to their original state after perturbation as long as the perturbation is not so great that it exceeds the attractor's counteracting feedback mechanisms. An appropriate analogy for cancer might be similar to 'a straw that broke the camel's back'. The 'state' of the camel, i.e., standing and bearing the weight being loaded onto its back, continues until a straw, an exceedingly small change compared to all the luggage previously piled onto its back, causes the entire system (the camel) to collapse. All the physiological thresholds that the camel previously employed to bear additional weight (e.g., muscle strength, balance, heart rate, blood sugar levels) collapsed because the addition of that tiny straw perturbed the system beyond its 
Page 4 of 7

ability to recover. In dynamical systems theory, such a huge change stemming from a small perturbation is referred to as a 'bifurcation', which can lead to a completely new attractor. For the camel, the new attractor is rest. The legs no longer function in their normal way to help the camel stand up, the heart rate is not sufficient, and blood sugar is too low. Genetic mistakes cannot survive if the body is functioning at a state of healthy dynamic equilibrium. Multiple redundant biochemical and biophysical (e.g. Bioelectric) mechanisms regulate function at the molecular, cellular and systemic levels to coordinate appropriate DNA repair, apoptosis, immune function, tissue repair and tumour suppression needed to destroy mutated cells and restore order. That is why cancer is more appropriately referred to as a systemic rather than a molecular disease. Problems (cancers) arise when the healthy attractor is perturbed beyond a point where the body's allosteric load exceeds its ability to repair itself (e.g., through dysfunction that accompanies aging, ingestion of toxicants, or exposure to carcinogens). A new attractor, a tumour, then evolves to replace it. At this point physiological functions have changed to such an extent that they support the new attractor. These system wide changes explain why tumours are so adept bypassing targeted therapies as shown in Figure 1.

\section{Breakdown in Cohesion of Bioelectric and Biochemical Network Signal Processing Leads to Breakdown in Healthy Systems Attractors}

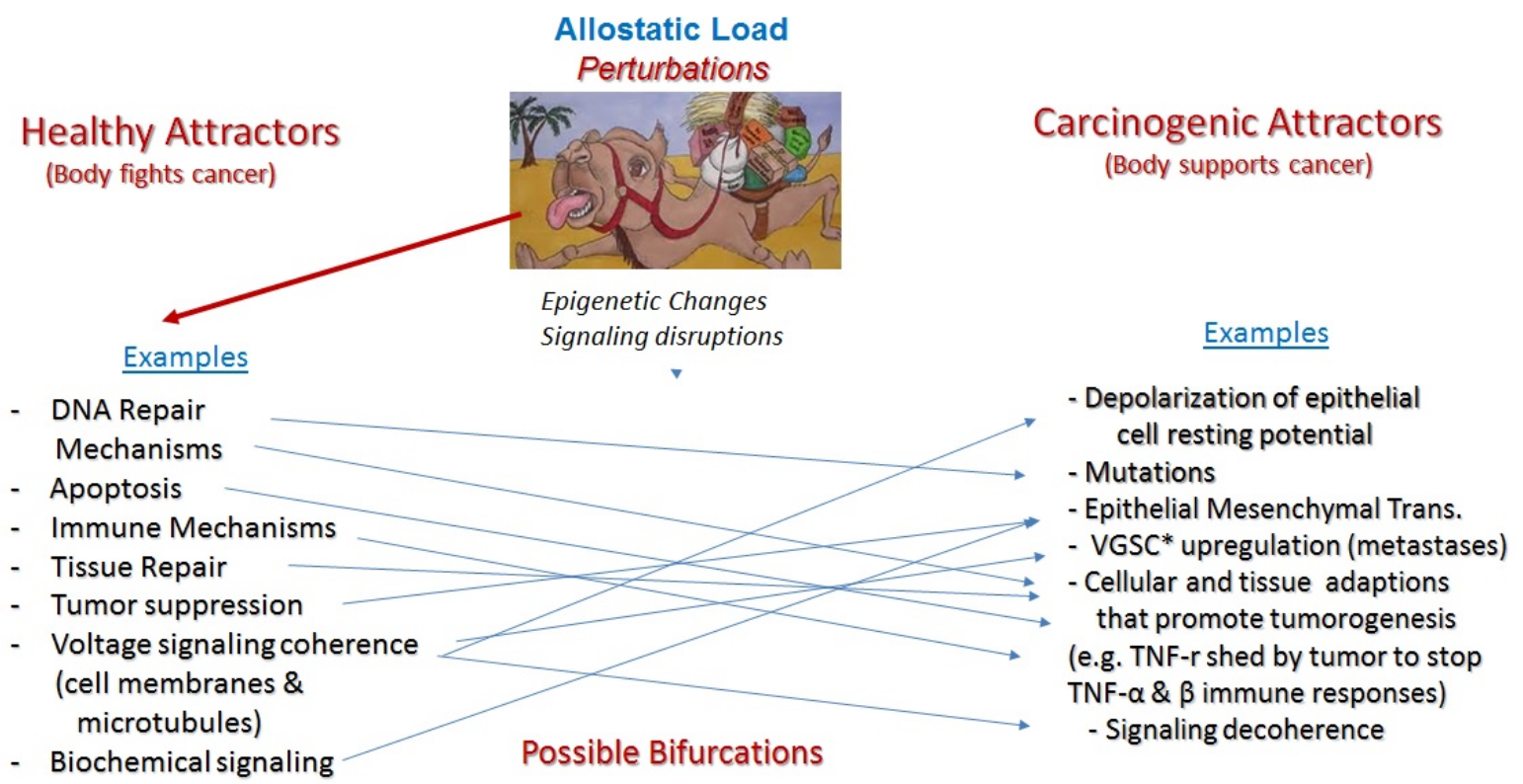

Figure 1: A bifurcation occurs when a small change made to the parameter values of a system causes a sudden 'qualitative' change in its behaviour ('straw that broke the camel's back') [ ${ }^{\star} \mathrm{VGSC}=$ voltage gated sodium channels].

\section{Biophysical Signalling: A Gap in Oncology Research}

A primary gap in mainstream oncology research is integration of the role of biophysical signalling pathways. The intrinsic bioelectric characteristics of cells and DNA are seldom incorporated into study designs of either dysfunctional molecular and cellular signal transduction, or integrated systemic regulation.

In normal, healthy cells, the inside of the cell is negative with respect to the outside. Changes in membrane potential arise from combined actions of many channels and pumps that segregate ions across the cell surface [13]. When the flow of ions, such as an influx of $\mathrm{Na}+$, across the membrane reduces the potential difference between the inside and the outside of the cell, we say that the cell becomes depolarized. Hyperpolarization occurs when voltage across the membrane increases, due either to an efflux of positive (e.g. + ions) or an influx of negative ions such as Cl-. Depolarization is typical of cancer cells $[54,55]$ and it has been demonstrated that depolarization reduces the differentiated phenotype [56], suggesting a role for membrane potential in the epithelial/mesenchymal transition. Mechanistically, in both healthy and malignant cells, ligand and voltage gated channels have been implicated in cell cycle check points during mitosis [19,55]. Hyperpolarization and depolarization characterize different phases of cell cycle progression accompanied by changes in the influx and efflux of different ions $(\mathrm{Ca} 2+, \mathrm{Na}+\mathrm{Cl}-)$ [13]. Accumulation of $\mathrm{Ca} 2+$ into mitochondria regulates mitochondrial metabolism and causes transient depolarization of mitochondrial membrane potential [57]. Impaired mitochondrial $\mathrm{Ca} 2+$ uptake alters the spatiotemporal characteristics of cellular $(\mathrm{Ca} 2+)$ signalling and down-regulates mitochondrial metabolism [57]. Impaired or abnormal Ca2+ overload may also lead to pathological states associated with cell death [57]. It has long been known that an excess of dairy (calcium) intake is a risk factor for prostate cancer [58-59] and it has been assumed that this is due to a lowering of concentrations of dihydroxyviatmin D3 a hormone thought to protect against prostate cancer [58]. Data on the 
role of $\mathrm{Ca} 2+$ in cell signalling indicates that it might be important to also investigate the role calcium in bioelectric signalling related to prostate cancer [59].

Trans membrane voltage gradients control cell proliferation migration, differentiation, and orientation [13,60-64]. In fact, ion channels have been implicated in all the hallmarks of cancer, including self-sufficiency in growth signals, insensitivity to antigrowth signals, evasion of programmed cell death, limitless replicative potential, sustained angiogenesis and tissue invasion and metastasis [19]. Why is clinical oncology research not making use of this abundant evidence in clinical research paradigms?

Microtubules, the other source of endogenous voltage potentials, are the primary organizers of the cytoskeleton and the electro-dynamic fields they generate are involved in organization, transport of molecules, interactions and information transfer [65]. Their structure is nonlinear and extraordinarily elastic, utilizing energy supplied by hydrolysis of GTP to GDP [24-26]. Microtubules play an important role in cell cycle regulation $[65,66]$ and it has been suggested that this regulatory activity is organized by quantum coherence and quantum entanglement among microtubule based centrioles and mitotic spindles [67]. A review of the evidence [67] shows that cancer cells do not divide symmetrically but have asymmetrical and multipolar distributions that are coordinated in space and time by centrioles. In vitro experiments in cultured cells show that when centrosomes (which contain the centrioles) are removed from the nucleus, it can reestablish an organizing centre but cannot undergo cell division/mitosis [67]. The scope of the present article does not permit detailed description of microtubule function but they are ferromagnetic lattices composed of tubulins that can assume multiple different conformational states. Even though they exist at room temperature, the water in cells is ordered due to surface charges [68], which creates a more crystal-like structure [67]. Molecules in the ordered state have lower energy than those in a disordered state and they oscillate in a common rhythm called a coherence domain [69]. This creates the possibility that they may transiently function similarly to a BoseEinstein condensate but at room temperature, unifying the mitotic complex with quantum coherence [67]. This theory would account for the replication of the three dimensional centriole structural geometry that is unlike that of DNA replication where mRNA comes into direct contact and binds with its complement [67].

Data also indicate that microtubules are involved in cancer not only through their role in mitosis and cell cycle regulation but also through their association with mitochondria and the Warburg effect [67]. Microtubules and mitochondria co-localize and generation of electromagnetic fields by microtubules depends on the function of mitochondria that supply its energy. Production of ATP utilizes an electrochemical proton gradient across the inner membrane of the mitochondria and the mitochondria alter the fields around microtubules through proton transfer. Because microtubules play a primary role in cell division, any serious theory of carcinogenesis mandates a quantum mechanical approach to better understanding their role and interactions with surrounding organelles in the cell.

In addition, although there are two known generators of endogenous bioelectric fields in the body, interactions between (cell and mitochondrial) membrane potentials and those generated by microtubules are not well understood. Because of data suggest an important role for both of them in carcinogenesis, this should be a focus of future research. Their structural proximity to each other and the fact that they propagate as fields rather than linear pathways, suggests the importance of quantum approaches.

\section{Conclusion}

Cancer is not a cell centric phenomenon. As we move beyond this paradigm to a systems approach, it would make sense to change the clinical goal from one of killing cells to reprogramming them back to a healthy attractor. Killing cells is based on the concept of using antibiotics to kill bacteria. The difference is that bacteria are an outside invader, whereas tumour cells are part of one's own body. Killing to heal may not be the best approach. Data suggest that microenvironmental reprogramming through a combination of biochemical and bioelectric signalling may be the next step in making significant strides towards the reduction of cancer mortality.

\section{References}

1. Heisenberg W (1990) Natural law and the structure of matter. Across Frontiers. Ox Bow Press. 115.

2. Zeng L, Zang J, Zhang S, Hao Z, Dong J, et al. (2017) AFEAP clonig: A precise and efficient method for large DNA sequence assembly. BMC Biotchnol 17: 81.

3. Zeng F, Zhang Y, Zhang Z, Malik AA, Lin Y (2017) Multiple-site fragment deletion, insertion and substitution mutagenesis by modified overlap extension PCR. Biotechnology \& Biotechnological Equipment 31: 339-348.

4. Zeng F, Hao Z, Li P, Meng Y, Dong J, et al. (2017) A restriction-free method for gene reconstitution using two single-primer PCRs in parallel to generate compatible cohesive ends. BMC Biotchnol 17: 32 .

5. Sharma S, Kelly TK, Jones PA (2010) Epigenetics in cancer. Carcinogenesis 31: 27-36.

6. Feinberg AP, Koldobskiy MA, Gondor A (2016) Epigenetic modulators, modifiers and mediators in cancer aetiology and progression. Nat Rev Genet 17: 284-299.

7. Porath D, Bezyadin A, de Vries S, Dekker C (2000) Direct measurement of electrical transport through DNA molecules. Nature 403: 635-638.

8. Yu ZG, Song X (2001) Variable range hopping and electrical conductivity along the DNA double helix. Physical Review Letters 86: 6018-6021.

9. Fink HW, Schonenberger C (1999) Electrical conduction through DNA molecules. Nature 398: 407-410.

10. Tuszynski JA (2008) Molecular and cellular biophysics. Chapman \& Hall/ CRC. Boca Raton. 133.

11. Scheer E (2014) Molecular electronics: A DNA that conducts. Nat Nanotechnol 9: 960-961.

12. Rice JC, Allis CD (2001) Histone methylation versus histone acetylation: New insights into epigenetic regulation. Curr Opin Cell Biol 13: 263-273.

13. Blackiston DJ, McLaughlin KA, Levin M (2009) Bioelectric controls of cell proliferation: Ion channels, membrane voltage and the cell cycle. Cell Cycle 8: 3527-3536.

14. Chernet BT, Levin M (2013) Transmembrane voltage potential is an essential cellular parameter for the detection and control of tumor development in a Xenopus model. Dis Model Mech 6: 595-607.

15. McCaig, Colin D, Song B, Rajnicek AM (2009) Electrical dimensions in cell science. J Cell Science 122: 4267-4276.

16. Foletti A, Grmaldi S, Lisi A, Ledda M, Liboff AR (2013) Biolectromagnetic medicine: The role of resonance signaling. Electromagn Biol Med 32: 484-499.

17. Nuccitelli R (2003) Endogenous electric fields in embryos during development, regeneraqtion and wound healing. Radiat Prot Dosimetry 106: 375-383.

18. Zhao Y, Zhan Q (2012) Electric fields generated by synchronized oscillations of microtubules, centrosomes and chromosomes regulate the dynamics of mitosis and meiosis. Theor Biol Med Model 9:26. 
Page 6 of 7

19. Prevarskaya N, Skryma R, Shuba Y (2010) Ion channels and the hallmarks of cancer. Trends Mol Med 16: 107-119.

20. Kunzelmann K (2005) Ion channels and cancer. J Membr Biol 205: 159-173.

21. Laub KR, Witschas K, Bilcher A, Madsen SB, Lvckhoff A, et al. (2012) Comparing ion conductance recordings of synthetic lipid bilayers with cell membranes containing TRP channels. Biochimica et Biophysica Acta 1818: 1123-1134.

22. Bates E (2015) Ion channels in development and cancer. Annual Review of Cell \& Development 31: 231-247.

23. Friesen DE, Craddock JA, Kalra AP, Tuszynski JA (2015) Biological wires, communication systems, and implications for disease. BioSystems 127: 14-27.

24. Pokorny J (2004) Excitation of vibrations in microtubules in living cells. Bioelectrochemistry 63: 321-326.

25. Craddock TJ, Tuszynski JA, Priel A, Freedman H (2010) Microtubule ionic conduction and its implications for higher cognitive functions. J Integr Neurosci 9: 103-122.

26. Pokorny J (1999) Conditions for coherent vibrations in the cytoskeleton. Bioelectrochem and Bioenerg 48: 267-271.

27. Knox SS, Ochs M (2013) Implications of systemic dysfunction for the etiology of malignancy. Gene Regul Syst Bio 7: 11-22.

28. Alfarouk KO, Stock C-M, Taylor S, Walsh M, Mudathir AK et al. (2015) Resistance to cancer chemotherapy; failure in drug response from ADME to P-gp. Cancer Cell Inter 15: 71.

29. Kamb A, Wee S, Lengauer C (2007) Why is cancer drug discovery so difficult?. Nat Rev Drug Discov 6: 115-120.

30. Potter JD (2014) The failure of cancer chemoprevention. Carcinogenesis 35: 974-982.

31. Siegel RLL, Miller KD, Jemal A (2016) Cancer statistics. CA: A Cancer J for Clinicians 20: 7-30.

32. Plankar M, Jerman I, Krašovec R (2011) On the origin of cancer: Can we ignore coherence? Prog Biophys Mol Biol 106: 380-390.

33. Bianconi E, Piovesan A, Facchin F, Beraudi A, Casadei R, et al. (2013) An estimation of the number of cells in the human body. Ann Hum Biol 40: 463-471.

34. Hoeijmakers JHJ (2009) DNA damage, aging, and cancer. New England J Medicine 361: 1457-1458.

35. Iyama T, Wilson DM (2013) DNA repair mechanisms in dividing and non-dividing cells. DNA Repair (Amst) 12: 620-636.

36. Venitt S (1996) Mechanisms of spontaneous human cancers. Environ Health Perspect 3: 633-637.

37. Jackson AL, Loeb LA (2001) The contribution of endogenous sources of DNA damage to the multiple mutations in cancer. Mutat Res 477: 7-21.

38. Mitchell JR, Hoeijmakers JHJ, Niedernhofer LJ (2003) Divide and conquer: nucleotide excision repair battles cancer and aging. Current Opinion in Cell Biology 15: 232-240.

39. Feinberg A, Ohlsson R, Henikoff S (2006) The epigenetic progenitor origin of human cancer. Nature Review Genetics 7: 21-33.

40. Huang S, Ingber DE. (2006-2007) A non-genetic basis for cancer progression and metastasis: Self-organizing attractors in cell regulatory networks. Breast Dis 26: 27-54.

41. Wynn ML, Ventura AC, Sepulchre JA, Garcia HJ, Merajver SD (2011) Kinase inhibitors can produce off-target effects and activate linked pathways by retroactivity. BMC Systems Biology 5:156.

42. Macneil LT, Walhout AJ (2011) Gene regulatory networks and the role of robustness and stochasticity in the control of gene expression. Genome Res 21: 645-657.

43. Albert R (2005) Scale-free networks in cell biology. J Cell Sci 118: 4947-4957.

44. Huang S, Kauffman SA (2012) Complex gene regulatory networks from structure to biological observables: Cell fate determination. Computational Complexity 6: 527-560.
45. DeBerardinis RJ, Lum JJ, Hatzivassiliou G, Thompson CB (2008) The biology of cancer: metabolic reprogramming fuels cell growth and proliferation. Cell Metab 7: 11-20.

46. Weaver WM, Petersen OW, Wang F, Larabell CA, Briand P, et al. (1997) Reversion of the malignant phenotype of human breast cancer cells in three-dimensional culture and in vivo by integrin blocking antibodies. J Cell Biol 137: 231-245.

47. Abbott DE, Bailey CM, Postovit LM,. Seftor EA, Margaryan N, et al. (2007) The Epigenetic Influence of Tumor and Embryonic Microenvironments: How Different are They? Cancer Microenviron 7: 246-255.

48. Kenny PA, Bissell MJ (2003) Tumor reversion: Correction of malignant behavior by microenvironmental cues. Int J Cancer 107: 688-695.

49. Hornberg JJ, Bruggeman FJ, Westerhoff HV, Lankelma J (2006) Cancer: A Systems Biology disease. Biosystems 83: 81-90.

50. Huang S, Ernberg I, Kauffman S (2009) Cancer attractors: A systems view of tumors from a gene network dynamics and developmental perspective. Semin Cell Dev Biol 20: 869-876.

51. Du W, Elemento O (2015) Cancer systems biology: Embracing complexity to develop better anticancer therapeutic strategies. Oncogene 34: 3215-325.

52. Knox SS (2010) From 'omics' to complex disease: A systems biology approach to gene-environment interactions. Cancer Cell Int 10: 11.

53. Chernet B, Levin M (2013) Endogenous voltage potentials and the microenvironment: Bioelectric signals that reveal, induce and normalize cancer. J Clin Exp Oncol S1-002.

54. Marino AA, Iliev IG, Schwalke MA, Gonzalez E, Marler KC, et al (1994) Association between cell membrane potential and breast cancer. Tumour Biol 15: 82-89.

55. Sundelacruz S, Levin M, Kaplan DL (2013) Depolarization alters phenotype, maintains plasticity of predifferentiated mesenchymal stem cells. Tissue Eng Part A 19: 1889-1908.

56. Duchen MR (2000) Mitochondria and calcium: From cell signalling to cell death. J Physiol 1: 57-68.

57. Chan JM, Stampfer MJ, Ma J, Gann PH, Gaziano JM, et al (2001) Dairy products, calcium and prostate cancer risk in the physicians' health study. Am J Clin Nutr 74:549-554.

58. Aune D, Rosenblatt DAN, Chan DSM, Vieira AR, Vieira R, et al (2015) Dairy products, calcium, and prostate cancer risk: A systematic review and meta-analysis of cohort studies. Am J Clin Nutr 101: 87-117.

59. Lobikin M, Chernet B, Lobo D, Levin M (2012) Resting potential, oncogene-induced tumorigenesis and metastasis: The bioelectric basis of cancer in vivo. Phys Biol 9: 065002.

60. Chernete BT, Levin M (2014) Transmembrane voltage potential of somatic cells controls oncogene-mediated tumorigenesis at long-range. Oncotarget 5: 3287-3306.

61. Levin M, Pezzulo G, Finkelstein JM (2017) Endogenous bioelectric signaling networks: Exploiting voltage gradients for control of growth and form. Annu Rev Biomed Eng 19: 353-387.

62. Levin M (2014) Endogenous bioelectrical networks store non-genetic patterning information during development an regeneration. J Physiology 592: 2295-2305.

63. Chernet BT, Fields C, Levin M (2015) Long-range gap junctional signaling controls oncogene-mediated tumorigenesis in Xenopus laevis embryos. Front Physiol 5: 519.

64. Cassimeris L (1999) Accessory protein regulation of microtubule dynamics throughout the cell cycle. Curr Opin Cell Biol 11: 134-141.

65. Westermann S, Weber K (2003) Post-translational modifications regulate microtubule function. Nat Rev Mol Cell Biol 4: 938-947.

66. Hameroff SR (2004)A new theory of the origin of cancer: quantum coherent entanglement, centrioles, mitosis, and differentiation. Biosystems 77: 119-136.

67. http://cdn.shopify.com/s/files/1/0161/7154/files/ FOURTH_PHASE_SAMPLE.pdf? 1585 
Citation: Knox SS (2018) Wave/Particle Duality and Biomedical Research Designs. Mol Bio 7: 215. doi:10.4172/2168-9547.1000215

Page 7 of 7

68. Pokorny J, Pokorny J, Borodavka F (2017) Warburg effect-damping of electromagnetic oscillations. Electromagn Biol Med 36: 270-278.
69. Pokorný J, Foletti A, Kobilková J, Jandová A, Vrba J, et al (2013) Biophysical insights into cancer transformation and treatment. Scientific World Journal 1-11. 